Methods Recognising an unmet educational need, a 'psychiatry in palliative care' study day was provided in 2018 (78 delegates) and 2019 (121 delegates), both were oversubscribed. All delegates were asked to complete an anonymous online questionnaire rating each topic, describing what was most and least useful; and how likely they would be to attend subsequent study days (completion rate $69 / 78$ in 2018, 84/121 in 2019).

Results Delegates were from a variety of healthcare backgrounds, disciplines and geographical regions with differing training levels. Across both years, reflecting learning needs, topics highly commended as extremely or very useful included 'personality disorder' (77/84, 91.6\%), 'treating delirium and dementia in palliative care' $(65 / 84,77.3 \%)$ and 'depression and psychosis in palliative care' (57/69 82.6\%). 142/153 $(92.8 \%)$ of participants across both days were extremely/very likely to attend subsequent events and/or recommend to a colleague. Qualitative feedback endorsed the value of interactive sessions and sharing learning about practical care and relationship building for service development. The event prompted development of a special interest module for psychiatry trainees embedded in palliative care teams, which is now in its second year, and sharing of audit tools to assess levels of combined palliative and psychiatric patient needs within palliative care services.

Conclusion These were important national learning events for best practice. We will continue, increasing capacity for 2020 . Our next study day will focus on latest advances in research, advice on setting up mental health support and risk assessment in the palliative care setting.

\section{TIME IS NOT AN EXCUSE, ADVANCE CARE PLANNING - THE USE OF A BRIEF INTERVENTION}

Catherine Hughes, Gill Horne, Wendi Abraham, Amanda Nadin, Kerry Macnish. Rowcroft Hospice, Torbay in partnership with Macmillan

\subsection{6/spcare-2020-PCC.48}

Background Lack of time is a recognised barrier to Advance Care Planning (ACP). ACP is a conversation that allows the person to specify preferences in relation to end of life care, stating wishes and choices.

Rowcroft Hospice with funding from Health Education England worked with people from different care settings to develop a brief intervention in ACP with the aim of openingup conversations with patients, giving staff prompts to help guide them.

Method Through a Macmillan funded project, we tested the Advance Care Planning Brief Intervention (ACP-BI) in practice for:

1. Usability by health care professionals (HCPs)

2. Acceptability by patients as reported by HCPs

3. To refine the training programme and BI, based on staff feedback

A steering group provided direction to the project. 24 HCPs were recruited from Nursing homes, Acute hospital, Community Hospitals and Community Nursing teams. A selfassessment tool was developed for the HCPs to test the intervention. Bespoke training was delivered and evaluated. Data on staff learning, including a reflective piece, were collected via a learning management system. Focus groups with HCPs were held midway and at the end of the project.

Results Not every person will engage with these conversations, HCPs included. However, the use of an ACP-BI, with training, and mentorship gives HCPs a building block to broach conversations about future planning, focusing on the patient's future and wishes. Qualitative feedback from nurses suggests it is possible to use a brief intervention for ACP: 'The initial conversation was only 15 minutes. I am surprised at how short these very important conversations can be'.

Conclusion Given that the median time of the 47 conversations was 12.5 minutes, time can no longer be used as a barrier to these conversations. We recommend the ACP-BI is further tested through research.

\section{HOW PAIN-RATING SCALES WORK (AND DO NOT WORK) IN REAL LIFE PRACTICE - A SMALL CONVERSATION ANALYTIC STUDY OF FIVE VIDEO- RECORDED HOSPICE CONSULTATIONS}

Laura Jenkins, Ruth Parry. Loughborough University

\subsection{6/spcare-2020-PCC.49}

Background Assessing pain is a key palliative care task. Structured tools to assess pain intensity are commonly used, but patients report challenges in responding to these.

Aims The study aimed to analyse real-life episodes of pain scale use within hospice care, and develop communication training resources based on the findings.

Methods Pain scale use was identified in a dataset of videorecorded hospice consultations involving 37 patients and five doctors in a large UK hospice. The video data was subjected to Conversation Analysis - a direct observational approach to describing the challenges, structure and functioning of people's interactional behaviours.

Results Pain rating-scales were used in 5/37 consultations. We found that patients capitalise on scale use: taking it as an opportunity to communicate multiple aspects of pain - not just intensity. Video-clips exemplifying this are included within 'Real Talk' training materials (www.realtalktraining.co.uk), alongside learning points detailing how practitioners can support and encourage patients to do so. We found instances (2/ 5 ) of numerical scale misunderstandings. The experienced doctors we recorded handled these carefully. A video-clip demonstrating how a doctor and patient resolve a misunderstanding with caution and sensitivity is included in the Real Talk materials. Analysis-based learning points describe how practitioners can avoid resolving the misunderstanding in ways that imply the patient is to blame, and also consider how misunderstandings can take considerable time and interactional effort to resolve.

Conclusions Our analysis shows that pain rating-scales get used to report features besides their official target. Our findings directly underpin communication training resources for face-to-face training events - showing how professionals can support patients' maximal responses and how they can handle numerical rating misunderstandings in ways that avoid demeaning or disempowering patients. 